\title{
PRELIMINARY REPORT OF THE WORK OF THE SUBGROUP ON COORDINATE FRAMES AND ORIGINS
}

\author{
J. KOVALEVSKY \\ OCA/CERGA \\ Avenue Copernic \\ F - 06130 GRASSE
}

\section{PARTICIPANTS IN THE WORK OF THE SUBGROUP}

The membership of the subgroup on coordinate frames and origins included the following : V.K. Abalakin, S. Aokd, F. Arlas, C. Boucher, N. Capitaine, K. Johnston, J. Kovalevsky (chairman), C. Ma, I.I. Mueller, C.A. Murray, H. Schwan, C.A. Smith, C. de Vegt and $\mathrm{R}$. Wielen.

In addition, the following colleagues have contributed to the work : V.A. Brumberg, T. Damour, J. Dickey, M. Feissel, T. Fukushima, B. Guinot, T. Huang, M. Standish, J.G. Williams and B.X. Xu.

\section{SCOPE OF THE STUDY}

The subgroup on coordinate frames and origins of the IAU Working group on reference systems was formed by J.A. Hughes as a follow up of the resolution $\mathrm{C} 1$ adopted by the IAU General Assembly in Baltimore. In particular, its work was to propose a practical realization of this resolution and, in particular of its section 3 :

"The International Astronomical Union should adopt a celestial reference based upon a consistent set of coordinates for a sufficient number of sultable extragalactic objects when the required observational data have been successfully obtained and appropriately analyzed. This reference frame should be based upon a common, simultaneous discussion of the observations using agreed upon conventions. This reference frame is likely to be based, initially at least, exclusively upon radio astrometry. and transformations between this reference frame and the conventional celestial and terrestrial reference systems as well as the dynamical frame should be defined. The reference frame should be updated as required".

To this, J.A. Hughes added the task of considering the origin of the celestial reference frame, having in mind that this problem is, "a separable problem which can be addressed somewhat independently. For example, the origins of the coordinate frames used for celestial coordinates and the metering of the Earth's rotation need not, in principle, be identical".

In practice, the problem of the terrestrial reference frame was not considered because I judged that this is essentially a problem to be addressed first by IUGG. This has been confirmed by $C$. Boucher who will make a proposal to this body. The presence of geodesists on the subgroup was intended to ensure that the proposals for a celestial reference frame and origins would not be incompatible with what could be the terres- 
trial reference frame in the future.

During the course of the work of the subgroup, I also decided not to discuss the origin of the Earth's rotation reckoning, because it was linked to the definition we would adopt for the celestial reference frame. I underestimated the difficulty of reaching a consensus on this first problem, but it can now be taken up at this stage.

\section{TERMINOLOGY}

In this presentation, the following terminology will be used :

i) Ideal reference system : Theoretical principle on which the final reference frame is based.

Example 1 : the equations of motion of a set of celestial bodies should have no Coriolis or linear acceleration terms when written in the ideal reference system.

Example 2 : the ensemble of very distant bodies has no global rotation in the ideal reference system.

ii) Reference system : It identifies the physical system on which the ideal reference system definition is applied. The solar system together with the physical laws governing it (general relativity or Newtonian mechanics) corresponds to the first example above. For the second example, a certain number of quasars form the system with a recipe on how the "non-rotation" is obtained..

iii) Conventional reference system : In addition to the statements 1 and 2, parameters describing the physical system are assigned (and are therefore conventional).

Example : masses and initial conditions of motions in the first case; they are given in the system of fundamental constants. In the case of extragalactic objects, a list of such objects will be given. The definition of the coordinate axes must also be given.

iv) Conventional reference frame or, simply, reference frame : It is a set of fiducial points with their coordinates that materialize the conventional reference system. The origin and axes of coordinates may either be materialized by, or simply inferred from the coordinates of the fiducial points. The coordinates of a point is obtained by interpolating the coordinates of fiducial points.

\section{BACKGROUND OF THE REFERENCE FRAME DEFINITION}

Before presenting the report on the work of the subgroup, I believe that it is appropriate to analyze somewhat deeply the meaning of the IAU resolution and its consequences. To do this, let us first examine the original meaning and the evolution of the notion of celestial reference frames and systems.

The objective of a celestial reference frame is to provide a means of assigning. in a unique way, coordinates of a celestial body, whether observed by an instrument, or derived from some theory. This can be - and has been - done by different methods. Let us examine them.

\subsection{Definition of a system of coordinates}

The most direct answer to the problem is to construct a system of rectangular or spherical coordinates in space. This is possible since there exists an ensemble of material bodies - Sun, planets and satellites - whose motions can be described by a theory referred to some cartesian coordinate system. The observed coordinates of these bodies are 
moving markers of the coordinate system. It is then sufficient to define an origin and a scale. The vernal equinox at a given date, and the mean equator at the same date, are a possible choice together with the astronomical unit of distance. Together with an absolute time, we obtain a self consistent definition of a dynamical reference system in the Newtonian sense.

Unfortunately, this not sufficient. We have no practical way of assigning the coordinates to $\alpha$ Centauri from a direct comparison of its position with the observed positions of one or several planets. This has to be done by a very complex procedure applied to a number of stars, so that one obtains a fundamental catalogue of stars, like the FK4. It is this catalogue that is used to determine the position of other bodies by relative astrometry. At this point, the user forgets completely the definition of the dynamical system, and only uses the frame represented by the catalogue.

\subsection{The case of the FK5}

As pointed out by $\mathrm{H}$. Schwan and R. Wielen, and in opposition to a common opinion (that I must confess to having shared), the FK5 system is not a dynamical system, but is essentially based on some determination of galactic rotation. Of course, this was ultimately linked to the position of the equinox point, in particular via a new constant of precession. But this is only an ad'hoc, a posteriori link.

So, although FK5 frame looks very much like the FK4 frame, the FK5 system is dramatically different. It is a kinematic reference system, based upon a certain model of galactic rotation. This choice was made because the observations of bodies in the solar system did not permit a sufficiently accurate definition of fixed points and a sufficiently accurate procedure for accessing these fixed coordinate axes.

\subsection{Accessibility of a position in a fixed coordinate system}

To obtain the coordinates of a body in the present reference system one requires a number of conventional parameters such as, the constant of precession, a theory of nutation, etc... that are to be applied in order to obtain its position in fixed coordinate system, chosen as being the position for J.2000.0 of the instantaneous mean equator and equinox. Any error in these conventional values introduces a spurious additional apparent motion of objects. This remark is particularly important when comparing the positions of quasars observed at several times and reduced to a given epoch by the present IAU conventional precession and nutation. This is equivalent, in the best cases, to a rotation of the system, more likely a rotation plus a deformation, the latter being due to systematic, position dependent errors in the proper motions.

The IAU resolution proposes a very elegant and easy solution for most of these problems. Let us, for clarity of exposition, assume that with an extragalactic objects are observable in the same wavelengths as the body with an unknown position. Since these objects are fixed, they materialize at any moment the reference system. and one will have directly the apparent position of the body in the coordinate system, without any use of precession or nutation correction. The reference system is directly accessed. This is a major simplification and corresponds exactly to what mathematically one means by a system of coordinates.

If the unknown body is observable only in visible light, one would interpolate its observation between observations of stars in a catalogue that is constructed with respect to the extragalactic frame defined beforehand. The positions of stars will be given at any epoch using a position at some date and proper motions. They will constitute an intermediary catalogue that may be continuously updated and improved, but it will be possible at any moment, and in any direction of the sky to link them with the actual extragalactic reference, since they will always be avallable as are presently 
avallable FK5 stars.

\subsection{Four dimensional reference system}

One of the drawbacks of the present FK5 reference system is that it is two dimensional, giving two angular parameters of position. In this sense it is not complete and it has to be completed by dimensional units. This is done by introducing a decoupled system of astronomical constants. This is no longer acceptable, and one should think of four dimensional reference system since it is now impossible to ignore General Relativity. However, a complete consistency in the four dimensions would have the drawbacks described in section 4.1. Therefore in some ways, one has to accept a certain decoupling. The proposal made later in this paper reduces this decoupling to a minimum since it imposes only SI units for time and length.

\section{DEVELOPMIENT OF THE WORK}

The work of the subgroup was done mostly by correspondence, although I had the privilege of direct discussions with about half of the members. In addition, I took the advice of several persons who were not members of the subgroup. After my first introductory letter sent on July 27, 1989, I prepared three others, taking into account the letters received previously. These answers posed, in the beginning. more questions and put forward more difficulties than they solved, so that it was not possible to test a first definition before April of this year. The present report is essentially based on the proposals included in my fourth letter and on the answers recelved thereafter. However, before entering into this presentation, it is useful to briefly describe some of the problems raised in the first part of the work.

\subsection{Structure of the reference system}

From answers to my first letter, it appeared that several members did not accept the LAU resolution and insisted upon either keeping the present situation, or having a dynamical definition of the reference system based upon the dynamics of the solar system.

Concerning the second point of view, my challenge to formalize a strictly dynamical definition was not taken up, and nothing was proposed until an incomplete attempt by $\mathrm{S}$. Aoki was recelved. It will be presented and discussed during the meeting. However, dynamical aspects have indeed to be included in the definition and this was implemented later.

The conservative point of view corresponds to a concern that the reference system should be accessible to all observers and in particular, to optical astrometry. This objection however is waived by the first section of the resolution C1 of the IAU which reads :

"In order to avold a confusing proliferation of reference frames, the FK5 should be retained as the LAU reference at optical wavelengths for the present and immediate future".

It is clear that this will remain true until a satisfactory extension of the radio frame to optically observable objects is achieved. Taking this into account, the opposition to a "primary radio-frame" lost part of its supporters. For this reason, in the following parts of the work I considered this problem as settled. Another point of doubt is the possibility of reconciling the variety of catalogues of radio sources into a single system. Later results and some presentations at this colloquium should dispel the fears. 
But the problems that were encountered should not hide the fact that the majority of the members of the subgroup do agree with the necessity of shifting quickly to a definition of a celestial reference frame based upon a system represented, as far as directions are concerned, by extragalactic objects.

\subsection{Relativistic aspects}

Although all agreed that the definition of a celestial reference system should be expressed in a form compatible with General Relativity, there was almost no input from the members on how to express this. I am grateful to $T$. Damour who finally suggested the general structure of the wording which will be presented here. It has the advantage of being consistent with the definitions of time scales and of being sufficiently concise so as to permit subsequent additions to the expression for $\mathrm{ds}^{2}$ if required.

\subsection{Non-rotation of an extragalactic reference frame}

Some members of the subgroup expressed fears that the coordinate system derived from the positions of extragalactic radio sources such as quasars would have a rotation. Let me quote a couple of sentences from a letter by R. Wielen: "It is an observed fact (and not only a plausible assumption) that the universe does not rotate within the very small errors of measurements. A stringent upper limit for the rotation of the universe is much beyond our present needs in astrometry". I believe that this point should not bring any difficulty. However a definition depending on the coordinates of two sources would not fulfill the non-rotation condition because of the possible instability of the sources, and one needs a large number of sources to stabilize the global behaviour. Actually, even if a rotation of the Universe is discovered, it will be orders of magnitude smaller than the rotation of the FK5 system realized by the present IAU conventional constant of precession.

\subsection{Presentation of the provisional conclusions}

In my last letter to the members of the subgroup, dated July 17, I made a proposal that now has to be discussed by the Colloquium. It consists of two general draft resolutions that provide the basis not only of the celestial reference system, but also of the time scales: hence they are common to our subgroup and to the subgroup on time scales. Two other draft recommendations are intended to describe how, from these conceptual recommendations, one could achieve the construction of a reference frame.

\section{GENERAL RECOMMENDATION}

\subsection{Conceptual recommendation (GI)}

\section{.... considering}

that it is necessary to define in the framework of the General Relativity Theory several systems of space-time coordinates,

recommends

the four space-time coordinates $\left(x^{0}=c t, x^{1}, x^{2}, x^{3}\right)$ be selected in such a way that in each coordinate system centered at the barycenter of an ensemble of masses exerting the main action, the interval $d s^{2}$ be expressed at the minimum degree of approximation by

$$
\left.d s^{2}=-\left(1-\frac{2 U}{c^{2}}\right)\left(d x^{0}\right)^{2}+\left(1+\frac{2 U}{c^{2}}\right)\left[\left(d x^{1}\right)^{2}+\left(d x^{2}\right)^{2}+d x^{3}\right)^{2}\right]
$$


where $c$ is the velocity of light and $U$ the sum of the gravitational potentials of the above mentioned ensemble of masses and of a potential generated by the external bodies, the latter vanishing at the barycenter.

This recommendation explicitely introduces General Relativity as the theoretical background for the definition. Only two terms, common to several representations of the field are kept, neglecting those terms that are different but are presently beyond the reach of observations. This permits the use of any of the possible more elaborate representation compatible with the given terms and still remain in conformity with the definition. It assumes the relevant PPN parameters to be equal to their values in General Relativity proper and leaves the possibility to add higher order terms. Finally it may apply to any frame origin (geocentric, barycentric, etc...). All members of the subgroup agreed with this principle which actually also introduces dynamics in the definitions.

\subsection{Constraint recommendation (G2)}

... considering

a) the necessity to define a barycentric coordinate system centered on the barycenter of the solar system and a geocentric coordinate system, centered on the barycenter of the Earth,

b) the desirability that the coordinate systems be linked to the best physically realized references in space and time,

c) that the use of the International System of Units (SI) should be extended to outer space, without introduction of scaling factors depending on the coordinate system under consideration.

recommends that

1. the state of rotation of the space coordinate grid centered at the solar system barycenter be such that the coordinates of a set of distant extragalactic objects present no global rotation,

2. the time coordinates be derived from the geocentric coordinate time realized by atomic clocks operating in conformity with the definition of the second.

3. the physical basic units of the space-time be the second of SI for the proper time and that it be connected to the meter of SI for proper length by the value of the velocity of light $c=299792458 \mathrm{~ms}^{-1}$.

This recommendation is the one that actually defines the characteristics of the reference systems. It fixes the state of rotation of the system (but its realization will have. of course, a certain amount of arbitrariness). To clarify the first point, one might state that the set should be large enough (not only two objects). The second part of the recommendation is to be discussed by the subgroup on time. It is to be noticed that these two recommendations exclude the use of scaling factors for the units of length and time.

T. Fukushima proposes to add that there should be no secular rotation among the space grids for coordinate systems to be used. I am not sure that this is compatible with 1 as applied directly to barycentric and geocentric frames. This is not true for a more general expression of the $\mathrm{ds}^{2}$. If it is true for the present case, it does add anything.

A. Murray suggests that this recommendation should include that the reference frame is defined by the ephemeris which have been derived from the relativistic equations of motion. I do not agree with this. The definition as given here plus a set of positions of quasars as implied by (1) is sufficient. The proposed addition would introduce internal inconsistencies. 
Most of the correspondents agreed with this wording. S. Aoki however, presented a drastically different proposal. It will not be discussed here since it is described elsewhere in this volume. A fear was expressed by J. Dickey and M. Standish who state that the introduction of a new unit of barycentric coordinate time that would not have the presently agreed scaling factor would introduce large corrections to the present ephemerides amounting to 2".0 per century for the Earth's orbit and 27".0 per year In the case of the Moon. They think that this would cause serious mistakes among users. However, not to reduce this scaling factor to one, would dramatically change the whole philosophy of the proposal and introduce inconsistency in the realization of the ideal system as proposed in G1.

\section{CONVENTIONAL REFERENCE SYSTEM}

Recommendations G1 and G2 set up the working rules for further developments in the construction of usable reference frames. The first step is to model the structure chosen to define the reference system. This means constructing the conventional reference system. Draft recommendation $\mathrm{R} 1$ proposes a route to do so. It reads :

\section{.... considering}

- the destrability to implement a unique conventional celestial barycentric reference system based upon the observed positions of extragalactic radio-sources,

noting

- the existence of lentative reference frames constructed by IERS and other Institutes, recommends

- that intercomparisons of these frames be extensively made in order to assess their accuracy and systematic differences,

- that IERS, in consultation will all the Institutes constructing catalogues of extragalactic radio-sources, establish a list of candidates for primary sources defining the new conventional reference system, together with a list of substitute sources that may later be added to or replace some of the primary sources. requests

- that such a list be presented to the 1994 IAU General Assembly for a decision on the definition of the new conventional reference frame,

- that onwards, the objects in this list be systematically observed by all VLBI astrometric programes.

The object of this recommendation is to request that the work on the implementation of the next barycentric reference frame consistent with G1 and G2 be started. It actually addresses the choice of the "set of distant extragalactic objects" that are mentionned in G2, first point.

This point has encountered one objection: the fact that IERS is requested to establish the list of objects. A strong request is that this should be the responsibility of an ad'hoc IAU Working Group. I would agree to this, provided however that it is shared with IERS and that the final compilation be made by IERS. It is not correct to say that IERS celestial reference frame is constructed for the rotation of the Earth. It has now more than 200 objects, only $10 \%$ of which are used for Earth's rotation. It is part of the tasks of IERS as defined by its bylaws to include the construction and the maintenance of a celestial reference frame and it has a sub-bureau that is devoted to this job. But $I$ admit that further inputs from astronomers having various interests in the choice would be valuable.

A more fundamental objection was made by C. Ma: IERS only combines the positions in existing catalogues and does not go into the actual raw data, so that the 
full strength of the underlying data is not used. This second approach is to be prefered to a simple concatenation. As a consequence, the work might have to be performed elsewhere in addition to the catalogue comparisons made by IERS which are as such very important to evaluate random and systematic errors of the catalogues.

Let me remark at this stage that at least one volce regretted that we do not adopt the existing IERS celestial reference frame.

An improvement of the wording has been requested by C.A. Murray: one should say more explicitely what is meant by "based upon" in the considering. I have no proposal at this point. It means that the catalogue that materializes the reference system is a set of coordinates of extragalactic radio-sources (just as the FK5 catalogue materializes the FK5 system).

\section{COORDINATE FRAME AND ORIGIN}

Once one has a catalogue of positions of extragalactic objects, one may apply to them an arbitrary rotation. Draft recommendation $\mathrm{R} 2$ makes a proposal and reads as follows :

\section{... considering}

- that the new conventional celestial barycentric reference frame should be as close as possible to the existing FK5 reference frame as refered to J.2000.0.

- that is should be accessible to astrometry in visual wavelengths as well as in radio wavelengths,

recommends

- that the positions of the extragalactic sources given tn the catalogue representing the reference frame be computed for the epoch J.2000.0 using the best avatlable values of precession and nutation.

- that a great effort be deployed in intercomparing reference frames of all types between them and particularly with FK5 and extragalactic reference frames,

- that all types of observing programs be undertaken or continued in order to link to a catalogue of extragalactic sources positions the best catalogues of star positions, in particular FK5 and the HIPPARCOS catalogues with the accuracy of these catalogues,

- that the $O x$ axis of the spherical coordinates of the new conventional celestial reference frames be as close as possible of the FK5, equinox J.2000.0 and that the principal plane be as close as possible to the mean equator at epoch J.2000.0.

This wording has brought a number of comments. The rationale for it was that one would like to avoid as much as possible a jump in positions and proper motions of stars and ensure the smoothest possible transition from the present situation. Clearly, it is not possible to have an alignment between FK5 and the new system other that at epoch. The proposed epoch is J.2000.0. The open questions are :

1. Should we use a conventional value of the precession to extrapolate the FK5 equinox or the continuously measured? The problem was not discussed throughly and should be an issue for the Colloquium: should one use as the origin at J.2000.0 the equinox and the mean equator as observed in year 2000 or extrapolate the FK5 value with the present IAU system of constants? The second solution is the easiest to implement, but the risk is that we shall not be at the actual mean equinox of date. I do not, feel very strongly in favour of my proposal in the recommendation, and most, but not all of the members of the subgroup that replied prefer using a conventional precession. An additional point is proposed by B. Guinot :

"That the new conventional celestial reference frame be not rotated to fit determination of the position of the mean pole of rotation and mean equinox for epoch 
J.2000.0".

This proposal implies in practice that we use a conventional value of precession. However, analysing the reasons of each side, one posstbly may find a common ground.

1) On one side (and this was the point I wanted to make in the proposal R2), it is only by using the best observed values of nutation and precession that observation of relative positions of radio-sources (extragalactic or radio-stars) can be compared and extrapolated to a reference date.

ii) On the other side, if one does this, the positions obtained will correspond to a position of the ecliptic and mean equator that is not the one of the FK5 system. In order to satisfy the last condition, on should then apply a rotation to the result obtained by (i) so that it corresponds to the FK5 mean pole and equinox at J.2000.0. It is to be noted that this double procedure is not equivalent to the procedure that consists to use systematically FK5 precession and nutation.

\section{TIMING AND TRANSITION PERIOD}

It is desirable that resolutions R1 and R2 are fulflled by 1994. This would permit linking HIPPARCOS to the extragalactic frame as well as to FK5 before it is published. Whether this would be sufficient to be ready with a stellar catalogue for the year 2000 Is difficult now to say, and whether would it be better than the present FK5 is still questionable. For this reason, I support the proposal made by $H$. Schwan which reads :

"As long as the relation between the optical and radio systems is not sufficiently accurately determined, the FK5 shall be considered as an additional realtzation of the celestial reference system in optical wavelengths".

Actually, it is simply a rephrasing of the first part of resolution $\mathrm{C} 1$ of the IAU (1988) quoted in 2.

\section{CONCLUSION}

The proposals made in this presentation are not perfect. Some improvements have been proposed that should be discussed during this Colloquium. There is also (S. Aokd) a completely different counter proposal that destroys most of the features of the proposed construction and in many ways reverts to the original situation. Science is advancing very quickly, and if IAU does not follow the trend, we shall arrive at an uncontrolable situation in which varlous interest groups will use different references without coordination. Actually, with the present considerable increase in the precision of observation, this temptation is already great for people working in VLBI and possibly also in space probes. It is time to take these improvements into consideration and the draft recommendations presented here are a tentative effort to meet their needs while they still remain acceptable to most classical astronomers. Then, of course, different realizations of the proposed system may be used for different usages or techniques, but all one of them, the conventional reference frame adopted, should be the standard to which other frames are to be identified as such as possible.

But this proposal has a more revolutionnary aspect which was perceived by some persons and perhaps made them more cautious. Reference frames have been until now based at least partially on the rotation and space motion of the Earth. The proposal removes all connection with the Earth. The Earth's motions become physical quantities that must be observed and studied for themselves but they lose the basic 
character of being the reference. There was a kind of geocentrism that our proposal destroys. Simllarly, the meter and the second become truly universal by means of relativistic transformations and will no longer be terrestrial units to which others are referred using rescaling. I believe that by adopting the ideas developed here, we would make a great step forward towards universality by throwing away the remainders of geocentrism. 